\title{
Single Quadrupole Multiple Reaction Monitoring Q-MRM enables Quantitative Mass Spectrometry
}

Jingchuan Xue $^{\dagger c}$, Rico J.E. Derks ${ }^{\bullet c}$, William Webb ${ }^{\dagger}$, Elizabeth M. Billings ${ }^{\dagger}$, Aries Aisporna ${ }^{\dagger}$, Martin Giera*•, and Gary Siuzdak*†§

†Scripps Center for Metabolomics, The Scripps Research Institute, 10550 North Torrey Pines Road, La Jolla, California 92037, United States

'Leiden University Medical Center, Center for Proteomics and Metabolomics, Albinusdreef 2, 2333ZA

Leiden, Netherlands

${ }^{\S}$ Departments of Chemistry, Molecular, and Computational Biology, The Scripps Research Institute, 10550

North Torrey Pines Road, La Jolla, CA 92037, United States

${ }^{\mathrm{c}}$ Co-first authors

*Corresponding authors: m.a.giera@lumc.nl and siuzdak@scripps.edu

TOC Image EISA Multiple Fragment lon Monitoring

with Single Quadrupole MS
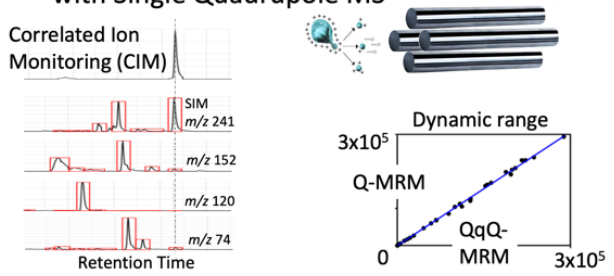

Abstract: A single quadrupole combined with enhanced in-source fragmentation/annotation (EISA) was used to perform multiple reaction monitoring (Q-MRM) for quantitative mass spectrometry analysis. EISA amplifies fragmentation of traditional soft electrospray ionization to produce fragment ions that have been found to be identical to those generated in tandem mass spectrometry. We have combined EISA fragmentation data with criteria established by the European Union Commission Directive 2002/657/EC for electron ionization single quadrupole quantitative analysis to perform quantitative Q-MRM experiments. These experiments were performed on multiple types of complex samples that included a mixture of 50 standards, as well as cell and plasma extracts. The dynamic range for Q-MRM quantitative analysis was comparable to triple quadrupole multiple reaction monitoring (QqQ-MRM) analyses at up to 5 orders of magnitude with the cell and plasma extracts showing similar matrix effects across both platforms. Amino acid and fatty acid measurements performed from certified NIST 1950 plasma with isotopically labelled standards demonstrated Q-MRM accuracy in the range of $91-110 \%$ for the amino acids, $76-129 \%$ for the fatty acids, and good precision (coefficient of variation $<10 \%$ ). In order to enhance specificity, a newly developed Correlated Ion Monitoring (CIM) algorithm was designed to facilitate these analyses. CIM autonomously processes, aligns, filters, and compiles multiple ions within one chromatogram enabling both precursor and in-source fragment ions to be correlated within a single chromatogram, also enabling the detection of co-eluting species based on precursor and fragment ion ratios. Q-MRM and CIM with single quadrupole instrumentation has been designed as an alternative to QqQ-MRM technology by correlating precursor and fragment ions to facilitate high sensitivity Q-MRM quantitative analysis on existing instrumentation that are generally inexpensive, easy to operate, and technically less complex. 


\section{INTRODUCTION}

Triple quadrupole multiple reaction monitoring (QqQ-MRM) mass spectrometry has existed since the $1970 \mathrm{~s}^{1,2}$, and with the advent of soft atmospheric pressure ionization in the late 1980s QqQ-MRM became the primary approach for targeted small molecule analysis ${ }^{3}$. In the last three decades QqQ-MRM has dominated the mass spectrometry landscape with its ultrahigh selectivity, sensitivity, and broad dynamic range, especially in the quantification of small molecules and peptides ${ }^{4-7}$. MRM exploits the unique capability of triple quadrupole (QqQ) mass spectrometers to act as a double mass filter, facilitating the analysis of analytes from complex matrices. In MRM mode, a predetermined precursor ion is selected/isolated with the first quadrupole, then fragmented in the collision cell (second quadrupole) with a neutral gas (e.g., nitrogen $)^{8}$. Thus, only precursor derived fragment ions pass to the third quadrupole and reach the detector. The precursor-fragment ion pairs are referred to as 'transitions' and over a hundred transitions can be recorded in (scheduled) MRM analyses, enabling the simultaneous targeted analysis of multiple analytes using liquid chromatography tandem mass spectrometry (LC-MS/MS)9 ${ }^{9}$ QqQ-MRM is considered the gold standard in the quantitative analysis of small molecules ${ }^{10}$ however, these instruments are typically expensive and suffer from inherent sensitivity losses in the collision chamber. Less expensive instrumentation, offering quantitative analysis for broader implementation, would provide a technically advantageous alternative option.

Three separate technologies have been combined and developed to provide quantitative analysis with single quadrupole multiple reaction monitoring (Q-MRM), they include: 1) soft atmospheric pressure ionization with enhanced in-source fragmentation/annotation (EISA), 2) multiple reaction monitoring, and 3) Correlated Ion Monitoring (CIM) algorithm. EISA is an approach that promotes the generation of both molecular ions and their respective fragments by increasing in-source fragmentation voltages. These fragment ions are the same as those generated in tandem mass spectrometry MRM experiments ${ }^{11-13}$. This similarity makes possible the simultaneous monitoring of both precursor ion and its fragments with a single quadrupole mass spectrometer, in experiments typically performed with tandem mass spectrometers (e.g., QqQ). To streamline the processing of the Q-MRM data, and flag potentially co-eluting species, a Correlated Ion Monitoring (CIM) algorithm was developed to facilitate these analyses. CIM autonomously processes, aligns, filters, and compiles multiple ions within one chromatogram enabling both precursor and in-source fragment ions to be correlated within a single chromatogram, also enabling the detection of co-eluting species based on precursor and fragment ion ratios.

The multiple fragment ion monitoring using a single quadrupole mass analyzer, is a variation on an approach that has been widely used for high sensitivity detection and quantitative analysis of small molecules especially with gas chromatography mass spectrometry (GC/MS) ${ }^{14}$. However, the Q-MRM combination of EISA and multiple reaction monitoring in a single quadrupole LC/MS instrument provides 
enhanced selectivity, specificity, and sensitivity for the quantitative analyses of a broader range of compounds (over traditional GC/MS). In addition, as compared with QqQ-MRM, Q-MRM is available at $\sim 30 \%$ the cost and have enhanced signal since the single quadrupole instruments do not have a collision cell and therefore do not experience the associated collision cell ion losses.

In this work, we demonstrate that EISA multiple fragment ion monitoring and CIM, performed with a single quadrupole mass spectrometer can have comparable quantitative performance with QqQ-MRM carried out using a triple quadrupole mass spectrometer. Using a mixture of endogenous molecules, we evaluated key analytical merits in quantitative analysis including selectivity, sensitivity/dynamic range, matrix effects, accuracy and precision. Subsequently, we used both Q-MRM and QqQ-MRM with the same triple quadrupole mass spectrometer to determine the concentrations of metabolites in a plasma extract, a bacterial cell extract, and a mammalian cell extract and the results acquired using both methods were compared. To confirm the applicability of Q-MRM, we further performed the analysis of a NIST certified plasma sample using a single quadrupole mass spectrometer.

\section{EXPERIMENTAL SECTION}

Materials. A total of 50 endogenous molecules were selected to represent a broad range of physicochemical properties and chemical structures, including amino acids, lipids, and fatty acids. For the investigation of quantitative performance of Q-MRM including the limit of quantification (LOQ) and dynamic range, the fifty molecules were prepared at 9 concentrations spanning 7 orders of magnitude: $0.2 \mathrm{nM}, 0.5 \mathrm{nM}, 1 \mathrm{nM}, 10$ $\mathrm{nM}, 100 \mathrm{nM}, 1 \mu \mathrm{M}, 10 \mu \mathrm{M}, 100 \mu \mathrm{M}$, and $1 \mathrm{mM}$. Additional intermediate calibration standards were used when necessary, resulting in at least five calibration points. For investigating matrix effects in EISA multiple fragment ion monitoring analysis, plasma (both human male AB plasma and NIST SRM 1950 plasma), mixtures of two mammalian cell lines (VERO C1008 and L6 myocyte), and bacteria cells (Pantoea strain sp. MT058) isolated from the groundwater in Oak Ridge Reservation were used. Standards and both plasma samples were purchased from Sigma-Aldrich (St. Louis, MO). VERO C1008 cell line and L6 myocyte cell line were obtained from James E. Voss laboratory in the Scripps Research Institute (La Jolla, CA) and Olivia Osborn laboratory in the University of California San Diego, respectively. Bacteria cell pellets were shipped from Michael W. Adams laboratory at University of Georgia and Nitin S. Baliga laboratory in the Institute for Systems Biology.

Sample preparation. Plasma samples were prepared using protein precipitation for both matrix preparation and amino acids extraction ${ }^{17}$. Briefly, $400 \mu \mathrm{L}$ solvent (acetonitrile:methanol; 1:1) was added to $100 \mu \mathrm{L}$ plasma sample. After storage at $-20^{\circ} \mathrm{C}$ for 1 hour, the sample was sonicated on ice for $10 \mathrm{~min}$, followed by 
centrifugation at $13,000 \times \mathrm{g}$ at $4^{\circ} \mathrm{C}$ for $15 \mathrm{~min}$. The supernatant was collected and dried in a vacuum concentrator (LABCONCO) at $10{ }^{\circ} \mathrm{C}$ and reconstituted with $100 \mu \mathrm{L}$ acetonitrile:water (1:1). After sonication (10 min, on ice) and centrifugation $\left(13,000 \times \mathrm{g}, 4^{\circ} \mathrm{C}, 15 \mathrm{~min}\right)$, the supernatant was transferred to a LC-MS glass vial with inserts for instrumental injection and for preparing matrix matched calibration lines. For the extraction of total fatty acids from plasma sample, a mixture of hexane/isopropanol (3:2) was used and $0.3 \mathrm{M}$ $\mathrm{KOH}$ in $80 \%$ methanol was used for alkaline hydrolysis, with details documented elsewhere ${ }^{18-19}$.

Bacterial and mammalian cells were extracted using a solvent mixture of acetonitrile:methanol:water $(2: 2: 1)^{20}$. In brief, cell samples ( $\sim 1$ million cells in each tube) were sonicated on ice for 15 min after shockfreezing in liquid nitrogen and subsequent thawing at room temperature. The operation was repeated three times. The samples were then incubated at $-20^{\circ} \mathrm{C}$ for one hour allowing protein precipitation, followed by centrifugation at $13,000 \times \mathrm{g}$ at $4{ }^{\circ} \mathrm{C}$ for $15 \mathrm{~min}$. Using the same procedure as mentioned above, the supernatant was collected, dried and reconstituted with $50 \mu \mathrm{L}$ acetonitrile:water (1:1). After sonication (10 min, on ice) and centrifugation $\left(13,000 \times \mathrm{g}, 4^{\circ} \mathrm{C}, 15 \mathrm{~min}\right)$, the supernatant was transferred to a LC-MS glass vial with inserts for instrumental injection and for preparing matrix matched calibration lines.

Triple quadrupole mass spectrometry analysis. Q-MRM and QqQ-MRM experiments were carried out on a Waters Xevo TQ-XS triple quadrupole mass spectrometer (Milford, MA) to allow for consistent comparison without the variability associated with using different instruments. Collision gas flow on the triple quadrupole was turned off when running the EISA multiple fragment ion monitoring experiment and the first quadrupole was used for analysis. The analyses were performed in both positive and negative electrospray ionization modes, and the Q-MRM and QqQ-MRM transitions measured for each molecule are shown in Table S1. In the Q-MRM experiments, the relationship between cone voltage and peak intensity of each ion (precursor ion and fragments) was investigated individually, and the optimal cone voltage at which maximum peak intensity is achieved was selected for each ion. In the QqQ-MRM experiments, the optimal cone voltage of the precursor ion was determined for every corresponding transition, and the collision energy was optimized for fragment ion generation. A dwell time of $15 \mathrm{~ms}$ was used for each channel in Q-MRM and each transition in QqQ-MRM. The ion source desolvation temperature was set at $500^{\circ} \mathrm{C}$.

ACQUITY UPLC BEH C18 column $(2.1 \times 100$ mm, $1.7 \mu \mathrm{m}$, Waters $)$ and ACQUITY UPLC BEH Amide column $(2.1 \times 100 \mathrm{~mm}, 1.7 \mu \mathrm{m}$, Waters $)$ were used in the separation of metabolites in reverse phase and HILIC analysis, respectively. For the reversed phase analyses, metabolites were separated by gradient elution at a flow rate of $200 \mu \mathrm{L} / \mathrm{min}$ starting at $25 \%(\mathrm{v} / \mathrm{v}) \mathrm{B}$, held for $1 \mathrm{~min}$, increased to $99 \% \mathrm{~B}$ within 8 min, held for $3 \mathrm{~min}$, and reverted to $25 \% \mathrm{~B}$ at $12.1 \mathrm{~min}$, held for $2.9 \mathrm{~min}$, with a total run time of $15 \mathrm{~min}$. The mobile phases comprised acetonitrile:water (60:40) containing $0.1 \%$ formic acid and $1 \mathrm{mM}$ ammonium formate (A) and isopropanol:acetonitrile (90:10) containing $0.1 \%$ formic acid and $1 \mathrm{mM}$ ammonium formate 
(B). For the HILIC analysis, metabolites were separated by gradient elution at a flow rate of $250 \mu \mathrm{L} / \mathrm{min}$ starting at $5 \%(\mathrm{v} / \mathrm{v}) \mathrm{A}$, increased to $80 \% \mathrm{~A}$ within $8 \mathrm{~min}$, held for $0.5 \mathrm{~min}$, and reverted to $5 \% \mathrm{~A}$ at $8.6 \mathrm{~min}$, held for $3.9 \mathrm{~min}$, with a total run time of $12.5 \mathrm{~min}$. The mobile phases were comprised of water containing $10 \mathrm{mM}$ ammonium acetate and $0.1 \%$ formic acid (A) and water/acetonitrile (5:95) containing $0.1 \%$ formic $\operatorname{acid}(\mathrm{B})$.

Single quadrupole mass spectrometry analysis. Q-MRM analysis of 11 amino acids (positive mode) and 5 fatty acids (negative mode) as well as their respective isotope labelled standards was performed on an Agilent 6130 Quadrupole LC-MS (Santa Clara, CA). The ESI source parameters in both positive and negative modes were set as follows: drying gas flow $9 \mathrm{~L} / \mathrm{min}$, drying gas temperature $350{ }^{\circ} \mathrm{C}$, and nebulizer pressure $30 \mathrm{psig}$. Capillary voltage was set at $4000 \mathrm{~V}$ in positive mode and $3500 \mathrm{~V}$ in negative mode, respectively. The fragmentor voltage was optimized for each monitored ion and dwell time was $290 \mathrm{~ms}$ at each channel.

ACQUITY UPLC BEH C18 column $(2.1 \times 100 \mathrm{~mm}, 1.7 \mu \mathrm{m}$, Waters $)$ was used in the analysis of fatty acids, and ACQUITY UPLC BEH Amide column $(2.1 \times 100 \mathrm{~mm}, 1.7 \mu \mathrm{m}$, Waters $)$ was used in the analysis of amino acids. The gradient in fatty acid analysis was as follows: starting at $20 \%(\mathrm{v} / \mathrm{v}) \mathrm{B}$, held for 1 min, increased to $95 \% \mathrm{~B}$ within $12 \mathrm{~min}$, held for $1 \mathrm{~min}$, and reverted to $20 \% \mathrm{~B}$ at $14.5 \mathrm{~min}$, held for $3.5 \mathrm{~min}$, with a total run time of $18 \mathrm{~min}$. The gradient in amino acid analysis was as follows: starting at 10\% (v/v) A, held for $2 \mathrm{~min}$, increased to $70 \% \mathrm{~A}$ within $11 \mathrm{~min}$, and reverted to $10 \% \mathrm{~A}$ at $11.5 \mathrm{~min}$, held for $3.5 \mathrm{~min}$, with a total run time of $15 \mathrm{~min}$. The mobile phases used in fatty acid and amino acid analysis were the same as those used in reverse phase and HILIC analysis in the triple quadrupole mass spectrometry analysis, respectively. The mobile phase flow rate was set as $100 \mu \mathrm{L} / \mathrm{min}$ in the analysis of both fatty acids and amino acids.

Data analysis. Extracted ion chromatograms (EICs) were generated using both Q-MRM and QqQ-MRM and manually inspected with vendor specific software. Targeted ions were carefully selected using precursor/fragment ions and retention time. The peak intensity and area were recorded for either comparison or quantification purpose. Each molecule typically has four associated EICs (one precursor and three fragment ions) in Q-MRM, which corresponds to three EICs (three MRM transitions) in QqQ-MRM. The peak area from each EIC was used separately in both Q-MRM and QqQ-MRM and the selection of the quantifier ion was based on the linear dynamic range of each ion (or transition) and the concentration of target metabolites in the test sample.

For the Q-MRM and QqQ-MRM experiments, molecular standards of all 50 molecules $(10 \mu \mathrm{M})$ were injected into the same triple quadrupole mass spectrometer to compare peak intensities of the same fragment ions. Following these analyses, limits of quantification (LOQs) and the linear dynamic ranges (LDRs) of the 
50 molecules were investigated for each acquisition method using a total of 9 concentration levels. The LOQ was defined as the lowest analyte concentration in the calibration that can be quantitatively detected within acceptable accuracy (back calculated 70-130\%) and precision (coefficient of variation $(\mathrm{CV})<30 \%$ ). This approach for determining LOQs was used since the signal-to-noise levels were expected to be impacted by the background levels generally being higher for Q-MRM. Using the solvent-based calibration ( $1 / \mathrm{x}^{2}$ weighted) acquired with both methods, the concentrations of the fifty metabolites were determined in a bacteria cell extract; for metabolites not endogenously present in the bacteria cell extract, standards were spiked into the sample at varying concentrations ranging from $10 \mathrm{nM}$ to $100 \mu \mathrm{M}$ for quantitative performance evaluation. These metabolites were also analyzed from a plasma extract and a mammalian cell extract to investigate the performance of Q-MRM in different types of matrices. Every test sample was replicated five times and the averaged results were used. CV, calculated as the percent ratio (\%) between standard deviation of observed metabolite concentration divided by the mean measured concentration, was used to assess the precision of replicate analysis. It is worth noting that since we used solvent based calibration standards in determining the LOQs, the values reported in this manuscript only reflect the instrumental quantification limits.

The biological matrices mentioned above, including plasma, bacterial cell extract, and mammalian cell extract, were selected as typical biological matrices to investigate the matrix effect of Q-MRM. Based on the results, the 50 metabolites were classified into two groups: those endogenously present and those absent. For endogenous metabolites, standard addition method was used to investigate the matrix effects at seven concentration levels, namely $0.1,1,5,10,50,100$, and $200 \mu \mathrm{M}$. The matrix effect was further assessed by comparing the peak area of the analyte standard in solvent and matrix at the same concentration, defined as [(peak area in solvent/in matrix $) \times 100]$. For those metabolites not present or at low levels $(<1 \mathrm{nM})$ in the target matrix, matrix matched calibration lines were prepared at concentrations ranging from $10 \mathrm{nM}$ to 200 $\mu \mathrm{M}$ by spiking standards into the post-extraction samples, referred to as analyte free matrix method. The slopes of the calibration lines for the same set of standards generated in both solvent and matrix were compared to assess the matrix effect. Each standard was replicated five times and the averaged peak area or slope was used in the analysis.

The accuracy and precision of Q-MRM was further evaluated by measuring amino acids and fatty acids in a certified NIST SRM 1950 plasma sample using isotope dilution-based matrix matched calibration. The intra- and inter-day precision of the method was determined by replicate analyses $(n=5)$ : intra-day precision was estimated using five replicate injections performed on a single day; inter-day precision estimation was performed based on five replicate injections conducted in five consecutive days. The linearity of the calibration was confirmed by plotting the peak area ratio of each metabolite (area metabolite/area internal standard) against its concentration. The sample concentrations were calculated from the equation $\mathrm{y}=$ 
$m x+b$, as determined by weighted $\left(1 / x^{2}\right)$ linear regression of the calibration line. The accuracy of the method was expressed as $[($ mean observed concentration $) /($ certified/reference mean concentration $)] \times 100$.

Correlated Ion Monitoring (CIM): The CIM algorithm was developed to perform peak picking, alignment and data analysis (https:/github.com/ricoderks/eisaCIM). In a typical experiment, chromatograms for each individual molecule are created by (1) peak picking each SIM trace within a pre-set intensity threshold (using XCMS v3.12.0). Determining the threshold for each SIM trace is a crucial step which allows for filtering co-eluting peaks. In the next step (2) the SIM traces are aligned and grouped within a retention time window (typically 3-5 seconds) where trace groups are retained only if they contain a peak for all four SIM traces (precursor and three fragment ions). (3) the filtered SIM traces are aligned and a CIM chromatogram is created.

\section{RESULTS AND DISCUSSION}

\section{In-Source Fragments Generation in EISA multiple fragment ion monitoring}

In earlier work, we demonstrated that enhanced in-source fragmentation allowed to produce both high abundance precursor ions and fragment ions that were characteristic of tandem mass spectrometry data for the majority of molecules investigated, thus enabling untargeted metabolomic experiments with single mass analyzers. In this effort we extend Q-MRM as a quantitative technology platform demonstrating that QMRM can also facilitate the quantitative analysis of small molecules in a single quadrupole mass spectrometer by producing high abundant precursor ion and respective fragments simultaneously. This can significantly improve the applicability of a single quadrupole mass spectrometer (Figure 1a) in quantitative analysis, which typically only uses the precursor ion in selecting the target analyte.

To investigate the in-source fragments generation performance of Q-MRM quantitation method, we prepared a group of fifty molecules containing a variety of chemical structures including amino acids, sugars, fatty acids, and lipids (Table S1). Except for the fatty acids analyzed in the negative mode, all other molecules produced at least 3 fragments (Table S1). In-source fragmentation was performed with a triple quadrupole to enable direct comparison of single and triple quadrupole performance on the same instrumentation. For example, the cone voltage in the Waters triple quadrupole mass spectrometer was optimized for each ion including both precursor and fragment ions to acquire their own maximum peak intensities. With the increase of cone voltage, peak intensity of ions either increase or a relationship of reversed U shape curve is observed (Figure S1). In Q-MRM, the optimal cone voltage of each ion was used in preparing the quantitative analysis method to acquire the precursor ion and its fragments at their own peak intensities. 
EISA - Enhanced In-Source fragmentation Annotation

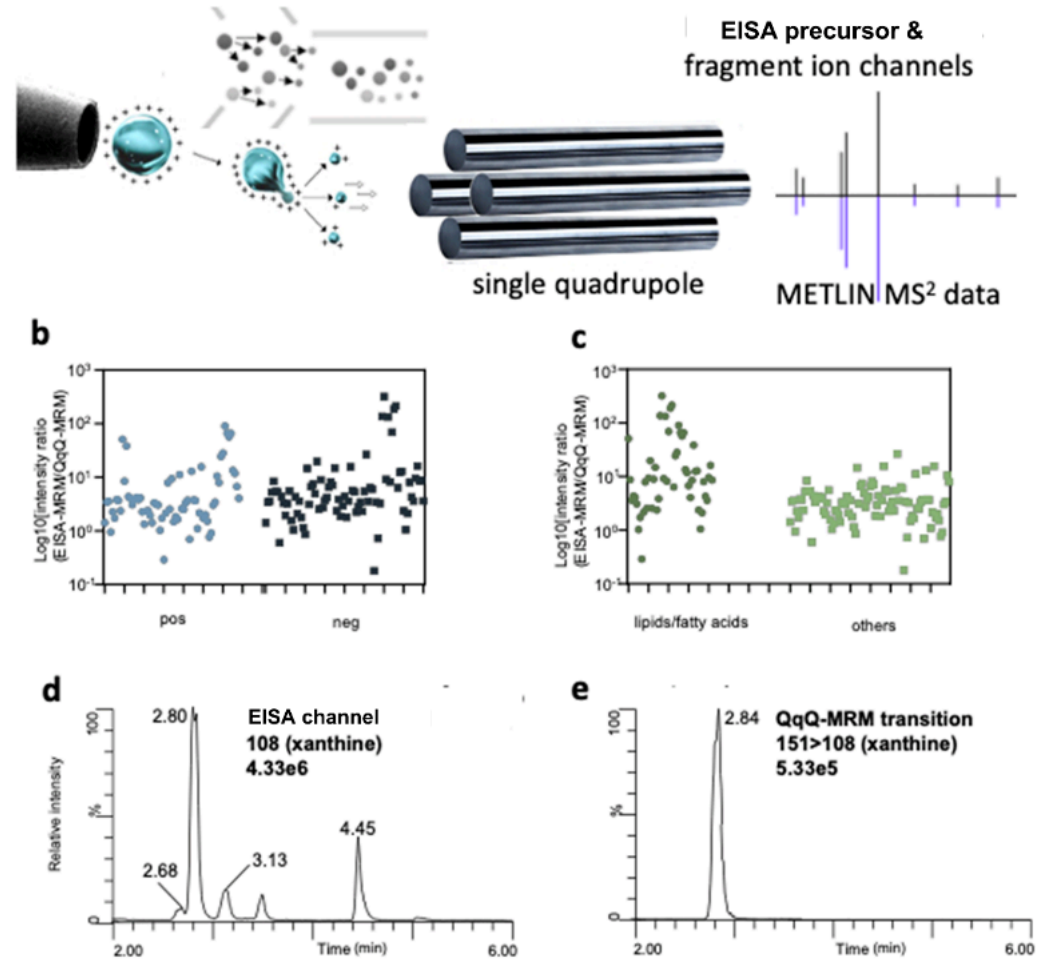

Figure 1. (a) General experimental design for Q-MRM with single quadrupole mass spectrometer. (b) Fragment ion intensity ratio (Q-MRM/QqQ-MRM) comparison between electrospray ionization positive mode and negative mode. (c) Fragment ion intensity ratio (Q-MRM/QqQ-MRM) comparison between lipids/fatty acids and other molecules. Log transformed intensity ratio was used in (b) and (c). (d) A typical extracted ion chromatogram of an Q-MRM channel (xanthine in solvent, fragment ion 108 at RT 2.80 min). (e) A typical extracted ion chromatogram of a QqQ-MRM transition (xanthine in solvent, MRM transition 151>108 at RT $2.84 \mathrm{~min}$ ). The number next to the Q-MRM channel or QqQ-MRM transition in (d) and (e) represents the maximum intensity of the EIC.

When performing the Q-MRM experiments on the triple quadrupole mass spectrometer, the collision gas flow was turned off and the first quadrupole was used for these analyses. In addition, for direct comparison purposes, we used QqQ-MRM to analyze the same collision-induced fragments generated in the collision cell for all the target molecules from the same instrument that the Q-MRM experiments were performed. Maximum intensity was optimized for each fragment ion by adjusting the relevant parameters including collision energy. Comparison of Q-MRM and QqQ-MRM chromatograms of identical standards (10 $\mu \mathrm{M}$ each) revealed that $86 \%$ of the fragments acquired using EISA had higher peak intensity with a median increase of around $300 \%$ and $400 \%$ in electrospray ionization positive and negative mode, respectively (Figure 1b), with an overall median increase of $260 \%$ in peak intensity (Figure S2). We further investigated the impact of Q-MRM on metabolites within different chemical classes and found that the median increase of fragment ion intensity for lipids and fatty acids were over $800 \%$, while it was over $300 \%$ for other molecules, indicating that Q-MRM provides different enhancements across different chemical classes (Figure 1c). For example, lipids and fatty acids appear to have enhanced fragmentation in Q-MRM. 
This analyte dependent fragmentation in Q-MRM can be further developed for optimizing quantitative analysis.

\section{Selectivity/Specificity}

Q-MRM would seemingly suffer from limited selectivity when compared to QqQ-MRM analysis, lacking the ability to generate transitional mass spectrometric pairs (as in QqQ-MRM) deriving from a selected precursor. However, Q-MRM takes advantage of its ability to simultaneously produce both high abundant precursor and fragment ions originating from the same molecule in a single quadrupole mass spectrometer. Therefore, by applying criteria originally adopted for GC/MS based selected ion monitoring quantitation, as described in EU Commission Directive 2002/657/EC ${ }^{15}$, we were able to generate quantitative data similar to what has been previously accomplished with GC/MS and QqQ-MRM. While QqQ-MRM exploits a tandem quadrupole mass analyzer's ability to selectively and simultaneously analyze both precursor and fragment ions resulting in four identification points (according to 2002/657/EC Directive), EISA multiple fragment ion monitoring implements this concept by monitoring one precursor ion and three respective fragments (termed "channels"). The resultant Q-MRM is composed of 4 channels, thereby equaling four identification points equivalent to two QqQ-MRM transitions with one precursor and two fragment ions, facilitating effective quantitative analysis. Xanthine is provided as an example, with a typical EIC from an EISA multiple fragment ion monitoring channel and a QqQ-MRM transition (using the same fragment ion) shown in Figure 1d and 1e, respectively.

The high specificity of QqQ-MRM in quantitative analysis is primarily achieved using specific precursor/fragment ion pairs and retention time. Single quadrupole MS analysis utilizes one mass filter and thus it is not possible to directly link fragments with the associated precursor ion. However, we observed that EISA can significantly improve the selective capability of target analytes from the $\mathrm{MS}^{1}$ spectra by simultaneously monitoring the precursor ion and its high abundant characteristic fragments $(\mathrm{n}=3)$ that elute at the same retention time. The in-source fragments serve as an identity indicator and can also be used for quantification once specificity is confirmed.

The METLIN small molecule retention time (SMRT) dataset ${ }^{21}$ and METLIN-MRM ${ }^{10}$ library created from the METLIN tandem mass spectra database ${ }^{22}$ has also been implemented to facilitate EISA multiple fragment ion monitoring (Figure 2) and help deconvolve commonly co-eluting compounds (Figure 2a). The METLIN SMRT dataset is an experimentally acquired reverse-phase chromatography retention time data set generated from over 80,000 small molecules. Using METLIN SMRT in conjunction with METLIN MRM offers an opportunity to help predict and deconvolve coeluting species. Leucine and isoleucine were analyzed as examples (Figure 2a). In addition, interfering molecular ions can be detected by comparing the peak intensity/area ratios between the precursor ion and its fragments (p:f1:f2:f3) across standards and 
samples (Figure 2b). This takes advantage of the fact that a peak intensity/area ratio between precursor ion and fragment ions stemming from one compound stay constant between standard and sample.

Another approach to enhance Q-MRM selectivity is through application of orthogonal separation technologies where the additional separate "channels" could be used for identification and/or quantification purposes. For example, ion mobility data could serve as an orthogonal set of information on the in-source fragment ions, as shown to be separated via drift tube-based ion mobility (Figure S3) and thus act as an alternative means for characterizing these ions in accordance with Directive 2002/657/EC ${ }^{15}$, using CCS values. This is demonstrated in Figure $\mathbf{S 3}$ where tandem mass spectrometry data is shown to coinciding with the in-source fragments where ion mobility could serve to provide additional discrimination between coeluting molecules and therefore further enhance specificity. Nevertheless, great care must be taken in choosing selective fragments ions for both, Q-MRM with CIM as well as MRM analysis as some analyte classes (e.g. fatty acids) tend to produce ubiquitous fragment ions (e.g. carboxylic acids predominantly show a loss of water and $\mathrm{CO}_{2}$ ).

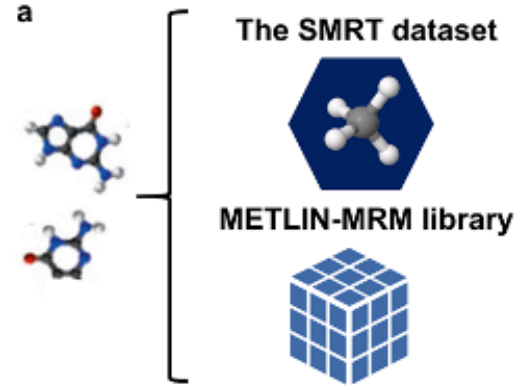

b

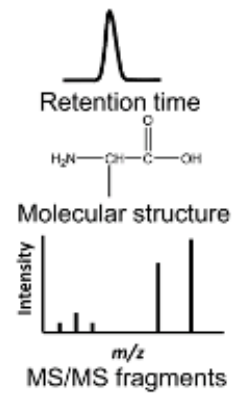

Peak intensity/area ratio comparison ( $p: f 1: f 2: f 3)$ standard

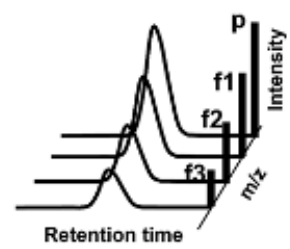

sample

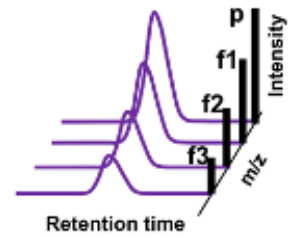

Characteristic fragments of coeluted metabolites

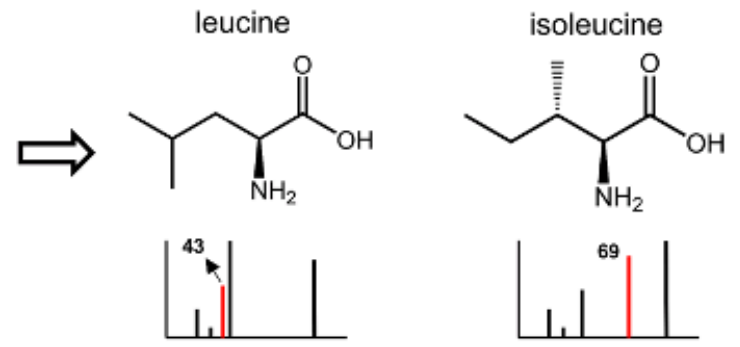

C

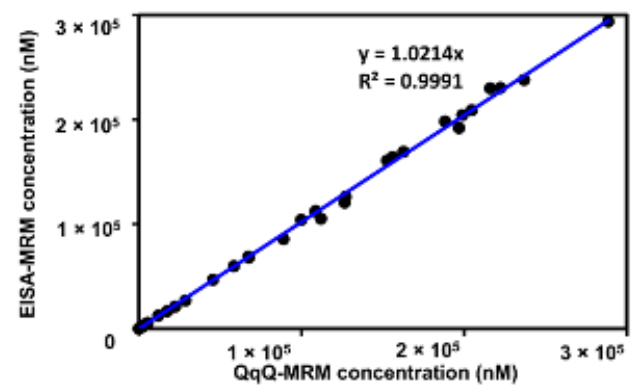

Figure 2. Characteristic fragments of co-eluting metabolites can be selected using retention time data generated from the SMRT dataset and fragmentation data from the METLIN-MRM library (e.g., (a) leucine and isoleucine). This data can be used to monitor the specificity of quantifier based on (b) peak intensity ratios, for example. Concentrations of 50 metabolites calculated using Q-MRM aligned well with the results calculated using QqQ-MRM generated from identical cell sample (analytical standards were spiked into the cell sample at varying concentrations if the metabolites were not detected in the cell sample) (c). p: precursor ion; f1, f2 and f3: fragment ion 1,2 , and 3 , respectively.

\section{Sensitivity/Dynamic Range}

A wide linear dynamic range (LDR) is important for small molecule quantitation in biological samples. According to the Human Metabolome Database, metabolite concentrations in human plasma and 
urine broadly vary from the pico- up to the millimolar range ${ }^{23,24}$. Having a wide LDR is useful in helping to avoid re-analysis and additional dilutions. The LDR can be limited either by the LOQ or by saturation at the ion source and detector ${ }^{25}$. Thus, we prepared solvent based calibrations for all fifty molecules at nine concentration levels ranging from $0.2 \mathrm{nM}$ to $1 \mathrm{mM}$ to compare the LDRs for the same compound between QMRM and QqQ-MRM ${ }^{16}$. Weighted $\left(1 / \mathrm{x}^{2}\right)$ linear regression calibrations were established for each molecule by plotting the relationship between concentration level and the corresponding peak area. LOQs were determined as the lowest concentration level in the calibration with acceptable accuracy and precision (Table S2), which were also used as the lower limit of the LDR. The upper limit in the LDR was determined using the same criterion. Here, only those molecules containing 3 fragments were analyzed and several molecules which didn't provide linear relationship between the concentration level and peak area were excluded. Finally, LDRs of a total of 43 molecules acquired using both Q-MRM and QqQ-MRM are reported (Table S3).

We first acquired the LDRs for each molecular ion (Q-MRM) or MRM transition (QqQ-MRM), then an integrated LDR was reported for each molecule in each method, combining the lower limit of the LDR of a primary sensitive ion and the upper limit of the LDR of a less sensitive ion. It was expected that sensitivity in Q-MRM was going to be lower compared to QqQ-MRM for the same molecule because of higher background noise levels observed in the EIC when only one mass filter is used. However, our results showed that Q-MRM can achieve the same sensitivity for most analytes in quantitative analysis. Of the 43 analytes, only $30 \%$ of the compounds tested had one order of magnitude lower LOQs in Q-MRM than QqQ-MRM. In addition, we observed that five molecules, including glucose, methylhistidine, glutathione, C20 sphingosine, and oleic acid, with LOQs one order of magnitude greater in Q-MRM, indicating that certain chemicals can be more efficiently detected in Q-MRM (Table S3). Thus, although there is a higher chemical noise baseline in the EIC produced by EISA, the majority of the compounds can provide the same LOQs as those produced using QqQ-MRM. Further, we found that $\sim 90 \%$ of the molecules had the same or higher upper limit of the LDR in EISA. This indicates that saturation was the same between the two methods for most ion signals and only $10 \%$ of molecules were saturated in Q-MRM. Overall, Q-MRM achieved an LDR of up to 5 orders of magnitude for over $90 \%$ of the analytes investigated.

Four molecules with different physicochemical properties, including valine, C20 sphingosine, glucose-6-phosphate, and eicosapentaenoic acid (EPA), were selected to illustrate the differences in LDR between the two techniques (Figure 3a and 3b). As shown in Figure 3c and 3d, EISA achieves similar or lower (C20 sphingosine) LOQs for the select compounds. Chromatograms of the four molecules at LOQ levels in solvent in both the Q-MRM and QqQ-MRM modes are shown in Figure 3e. As illustrated, while Q-MRM comes with a greater level of chemical noise, the higher ion intensity enables comparable sensitivity. Of the four molecules, valine and EPA presented an identical dynamic range between the two 
modes; Q-MRM analysis of C20 sphingosine proved more sensitive when compared to QqQ-MRM allowing to extend the dynamic range towards lower analyte concentrations; for glucose-6-phosphate, detector saturation was observed at $1 \mathrm{mM}$ in Q-MRM, while it was not observed in QqQ-MRM mode.

Chromatograms of the four molecules acquired using Q-MRM and QqQ-MRM modes in a bacteria cell extract are shown in Figure 3f. In summary, Q-MRM performs equally well or in some cases better in terms of sensitivity and dynamic range for most metabolites investigated, while QqQ-MRM mode was less prone to detector saturation.

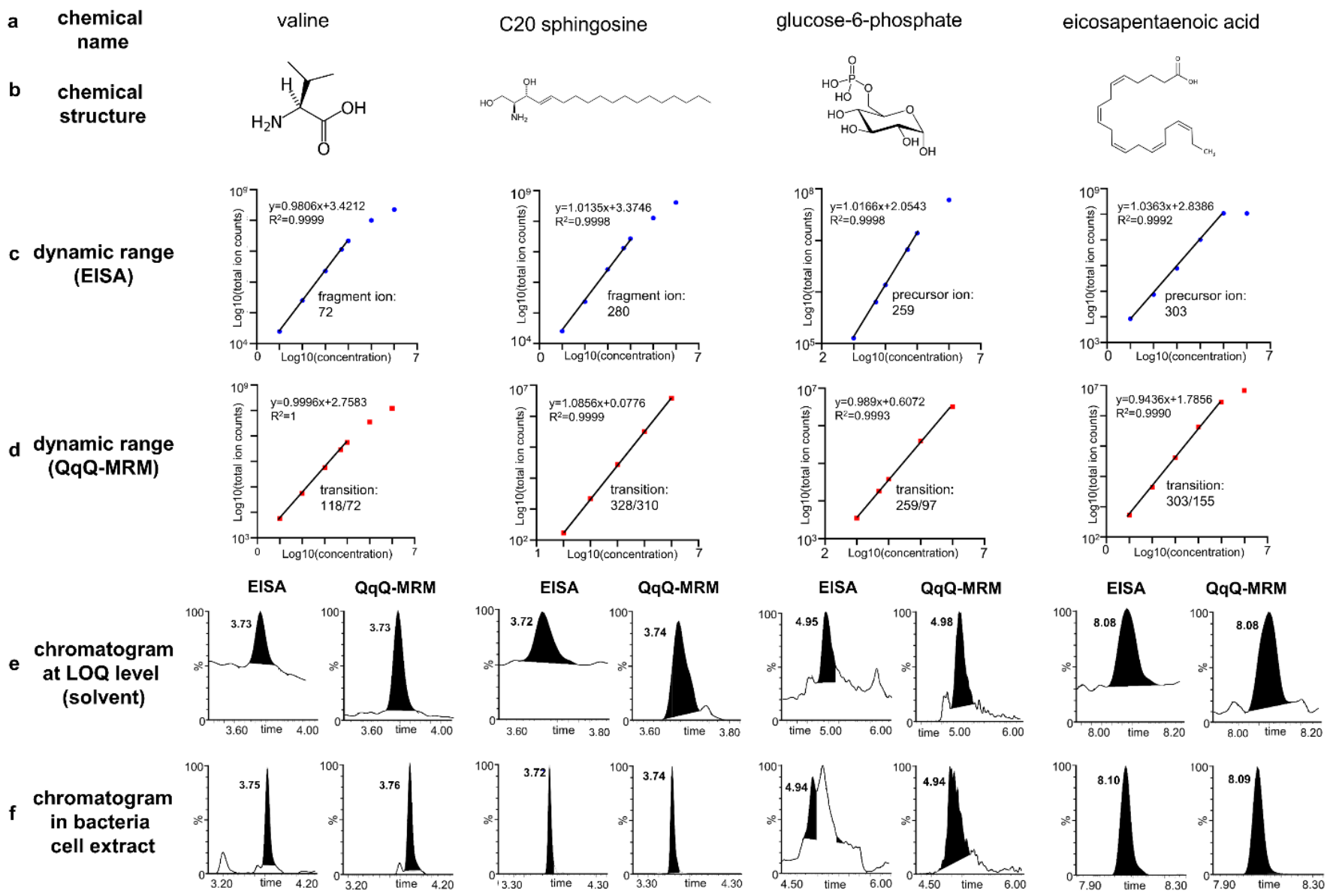

Figure 3. Chemical names (a) and structures (b), dynamic ranges (c, d), LC chromatograms at LOQ levels in solvent (e), and LC chromatograms in bacterial cell extracts (f) of four representative molecules with different physicochemical properties. Dynamic range data and LC chromatograms acquired in both Q-MRM and QqQMRM are shown; the linearity was calculated based on the log10 transformed values; the ion used with EISA and the transition used in QqQ-MRM are shown next to the linear curve. Valine and glucose-6-phosphate were acquired using HILIC chromatography; $\mathrm{C20}$ sphingosine and eicosapentaenoic acid were acquired using reverse phase chromatography.

\section{Application and comparison of Q-MRM and QqQ-MRM in cell extracts and plasma samples}

Additional analyses were performed using the above-mentioned calibration approaches to quantify these molecules from a bacteria cell extract using both Q-MRM and QqQ-MRM. For those compounds which were not endogenously present, the bacteria cell extract was fortified with a defined amount of 
metabolite standards for quantification purposes. The concentrations calculated ranged from $1 \mathrm{nM}$ to over $100 \mu \mathrm{M}$ levels and good alignment was observed between the results calculated using the two techniques (Figure 2c; Table S4). We further analyzed these metabolites in a plasma extract and a mammalian cell extract using both Q-MRM and QqQ-MRM, and concentrations of the detected metabolites are shown in Tables S5 and S6, respectively. The percent difference of the results calculated using the two techniques were below $7 \%$ for over $90 \%$ of the molecules measured in every matrix. Significantly co-eluting peaks from the matrix can interfere with the accuracy of peak integration and lead to a higher percent difference, e.g., uric acid in plasma.

Table 1. Slopes of calibration lines for inosine in plasma extract and glucose-6-phosphate in bacteria cell extract as well as their calibrations from solvent using both Q-MRM and QqQ-MRM quantification techniques.

\begin{tabular}{|c|c|c|c|c|c|c|}
\hline & \multicolumn{3}{|c|}{ QqQ-MRM } & \multicolumn{3}{|c|}{ Q-MRM } \\
\hline & solvent & $\begin{array}{c}\text { plasma extract } \\
\text { (inosine) }\end{array}$ & Mann-Whitney test & solvent & plasma extract (inosine) & $\begin{array}{c}\text { Mann-Whitney } \\
\text { test }\end{array}$ \\
\hline slope $^{a}$ & 195.4 & 197.1 & $p>0.01$ & 2356.2 & 2389.8 & $p>0.01$ \\
\hline$S D^{b}$ & 2.4 & 6.5 & & 46.8 & 109.3 & \\
\hline $\mathrm{CV}(\%)^{\mathrm{c}}$ & 1.2 & 3.3 & & 2 & 4.6 & \\
\hline & solvent & $\begin{array}{l}\text { bacteria cell (glucose- } \\
\text { 6-phosphate) }\end{array}$ & Mann-Whitney test & solvent & $\begin{array}{c}\text { bacteria cell (glucose-6- } \\
\text { phosphate) }\end{array}$ & $\begin{array}{c}\text { Mann-Whitney } \\
\text { test }\end{array}$ \\
\hline slope & 15 & 24.1 & $p<0.01$ & 200.4 & 381.2 & $p<0.01$ \\
\hline SD & 0.6 & 1.2 & & 2.4 & 5.2 & \\
\hline CV(\%) & 3.9 & 4.8 & & 1.2 & 1.4 & \\
\hline
\end{tabular}

${ }^{\mathrm{a}}$ slope, calculated using the linear calibration between total ion counts $(\mathrm{y})$ and concentration $(\mathrm{x})$ of the analyte; ${ }^{\mathrm{b}} \mathrm{SD}$, standard deviation between slopes $(n=5) ;{ }^{\circ} \mathrm{CV}$, coefficient of variation, calculated as the percent ratio between the standard deviation and mean value of the slope.

\section{Matrix Effect}

A key advantage of QqQ-MRM analysis of complex mixtures is the high selectivity achieved by the combination of two mass filters and a collision cell. As Q-MRM is performed with a single quadrupole, selectivity in complex mixture analysis is an important analytical feature that requires evaluation. Thus, we investigated three complex biological matrices, including a plasma extract, a mammalian cell extract, and a bacteria cell extract. As mentioned above, we first analyzed the three matrices and measured the concentrations of fifty metabolites in each of them. For those metabolites which were present in the matrices, we used the standard addition method to evaluate the matrix effects by spiking five to eight different concentration levels into the extract post-extraction ${ }^{16}$. For metabolites not endogenously present in the matrices, we spiked eight different concentration levels into each of the three different matrices postextraction. Matrix effects were evaluated according to Matuszewski et al. ${ }^{16}$ and as described below, for all fifty metabolites in both Q-MRM and QqQ-MRM. Overall, the two techniques showed similar matrix effect trends (suppression or enhancement) for the same metabolite and matrix. There were no significant differences of the matrix effects observed between Q-MRM and QqQ-MRM. Five molecules, including phenylalanine, hypoxanthine, uridine, inosine, and guanosine, were selected to demonstrate the matrix effects of molecules in the three different matrices assessed using the standard addition method (Tables S7- 
S9). We established calibration lines for metabolites which were not present in the sample and compared their slopes in solvent and matrix as well as the standard deviations in replicate injections $(n=5)$ with examples shown in Table 1. Overall, our results indicate that the difference of matrix effects between the two analytical modes, are compound and matrix dependent and independent of the analytical technique (QMRM versus QqQ-MRM).

\section{Accuracy/Precision of EISA Multiple Fragment Ion Monitoring of NIST Certified Plasma}

The accuracy and reproducibility (Table 2) were examined with a quantitative isotope dilution-based analysis method using Q-MRM on a single quadrupole mass spectrometer via the analysis of 11 amino acids and 5 fatty acids in a certified NIST 1950 plasma sample. Calibration lines were prepared using the ratio between external calibration standards and the corresponding isotopically labelled internal standards versus the response-dependent concentration factor (concentration of the target analyte divided by the concentration of the internal standard). The results were compared to the certified or reference values provided by NIST for accuracy analysis. Precision was evaluated as intra- and inter-day CV as described above in the data analysis section.

As shown in Table 2, the results acquired using Q-MRM demonstrated very good accuracy (91$110 \%$ for amino acids; $76-129 \%$ for fatty acids) and inter-day precision $(\mathrm{CV}<10 \%)$. The wider accuracy range for fatty acids may be partly ascribed to the different sample analysis protocol and instrument used by NIST (GC-MS). The LC (Agilent 1260 infinity) coupled with the single quadrupole mass spectrometer was not able to handle the high pressure, thus leucine and isoleucine were not distinguishable here. Therefore, a combined result for the two compounds was reported, which had a relative higher inter-day CV (8.7\%).

Table 2. Q-MRM analysis of 11 amino acids and 5 fatty acids in NIST SRM 1950 plasma.

\begin{tabular}{|c|c|c|c|c|c|c|c|}
\hline \multirow[b]{2}{*}{ metabolite } & \multirow{2}{*}{$\begin{array}{c}\text { certified/reference } \\
\text { concentration }^{\mathrm{a}} \\
(\mu \mathrm{M})\end{array}$} & \multicolumn{3}{|c|}{ intraday } & \multicolumn{3}{|c|}{ interday } \\
\hline & & $\begin{array}{c}\text { measured } \\
\text { concentration } \\
(\mu \mathrm{M})\end{array}$ & $\begin{array}{c}\text { accuracy } \\
(\%)\end{array}$ & $\begin{array}{l}C^{c} \\
(\%)\end{array}$ & $\begin{array}{c}\text { measured } \\
\text { concentration } \\
(\mu \mathrm{M})\end{array}$ & $\begin{array}{c}\text { accuracy }^{b} \\
(\%)\end{array}$ & $\begin{array}{l}C V^{c} \\
(\%)\end{array}$ \\
\hline histidine & $72.6 \pm 3.6$ & 67.9 & 93.6 & 1.1 & 66.2 & 91.2 & 2.1 \\
\hline leucine/isoleucine & $155.9 \pm 9.7$ & 164.2 & 105.3 & 5.6 & 160 & 102.6 & 8.7 \\
\hline lysine & $140 \pm 14$ & 134.5 & 96.1 & 0.4 & 136.7 & 97.6 & 0.8 \\
\hline methionine & $22.3 \pm 1.8$ & 22.2 & 99.7 & 1.4 & 21.3 & 95.5 & 2 \\
\hline proline & $177 \pm 9$ & 172.3 & 97.3 & 1.1 & 174.7 & 98.7 & 1.4 \\
\hline threonine & $119.5 \pm 6.1$ & 109.3 & 91.4 & 0.2 & 110.1 & 92.1 & 0.4 \\
\hline tyrosine & $57.3 \pm 3.0$ & 54.9 & 95.9 & 1.3 & 54.5 & 95.1 & 2.8 \\
\hline valine & $182.2 \pm 10.4$ & 181.3 & 99.5 & 0.6 & 181.8 & 99.8 & 0.7 \\
\hline phenylalanine & $51 \pm 7$ & 46.5 & 91.2 & 2.8 & 46.8 & 91.8 & 2.5 \\
\hline arginine & $81.4 \pm 2.3$ & 85.8 & 105.4 & 1.1 & 88 & 108.1 & 3.3 \\
\hline $\begin{array}{l}\text { eicosapentaenoic } \\
\text { acid }\end{array}$ & $38.6 \pm 0.5$ & 36.9 & 95.7 & 4.4 & 35.9 & 93 & 4.6 \\
\hline
\end{tabular}




\begin{tabular}{lccccccc} 
linoleic acid & $2838 \pm 143$ & 2081 & 73.3 & 4.9 & 2157.9 & 76 & 5.3 \\
oleic acid & $1614 \pm 154$ & 1450 & 89.9 & 3.8 & 1456 & 90.2 & 5.3 \\
myristic acid & $80.1 \pm 17.0$ & 98.6 & 123 & 3.6 & 98.3 & 122.7 & 3 \\
stearic acid & $644 \pm 41$ & 822 & 127 & 1.3 & 829.7 & 128.8 & 1.4 \\
\hline
\end{tabular}

${ }^{a}$ reference concentrations for arginine and phenylalanine, certified values for all others; ${ }^{b}$ accuracy was calculated as the percent ratio between measured concentration and certified/reference concentrations (mean) in the sample; ' $\mathrm{CV}$ : coefficient of variation, calculated as the percent ratio between standard deviation and mean values of the measured concentrations $(n=5)$.

\section{Correlated Ion Monitoring}

Q-MRM quantitative analysis as described above was validated using traditional SIM technology. However, distinct from multi-SIM type approaches and in order to enhance selectivity, a Correlated Ion Monitoring (CIM) algorithm was developed to autonomously process, align, correlate, and filter SIM data. The CIM algorithm is designed to align and selectively compile multiple ions within one chromatogram and flag signals if they fall outside manually preset thresholds. The filtering was incorporated to help detect coeluting molecules. The CIM chromatogram is created as a compilation of the individual ion signals only if each signal satisfies pre-set criteria. To accomplish this, CIM algorithm analyzes the SIM traces within a prespecified RT window from mzxml files. CIM then performs peak picking, alignment, threshold and ratio filtering with settings that can be adjusted to maximize performance.

The function of monitoring the ratio between the precursor and the fragment ions was incorporated to identify co-eluting peaks that have either the same precursor or fragment ions. Thus, if a particular ion is found not to be within the pre-determined expected ratio parameters, the measured molecule is either flagged for quantitative removal, or the particular ion is eliminated from the quantification analysis. This approach could also be used for QqQ, since co-eluting peaks can also impact QqQ quantitative accuracy. The CIM chromatogram represents a trace comparable to mass transitions obtained from a QqQ-MRM experiment as demonstrated here with the analysis of cystine from a cell extract and leucine/isoleucine from a plasma extract (Figure 4). Additionally, CIM generates reconstructed EISA fragment ion spectra allowing for the continuous monitoring of ion ratios and matching against authentic standard materials. In this way selectivity issues can autonomously be detected. 

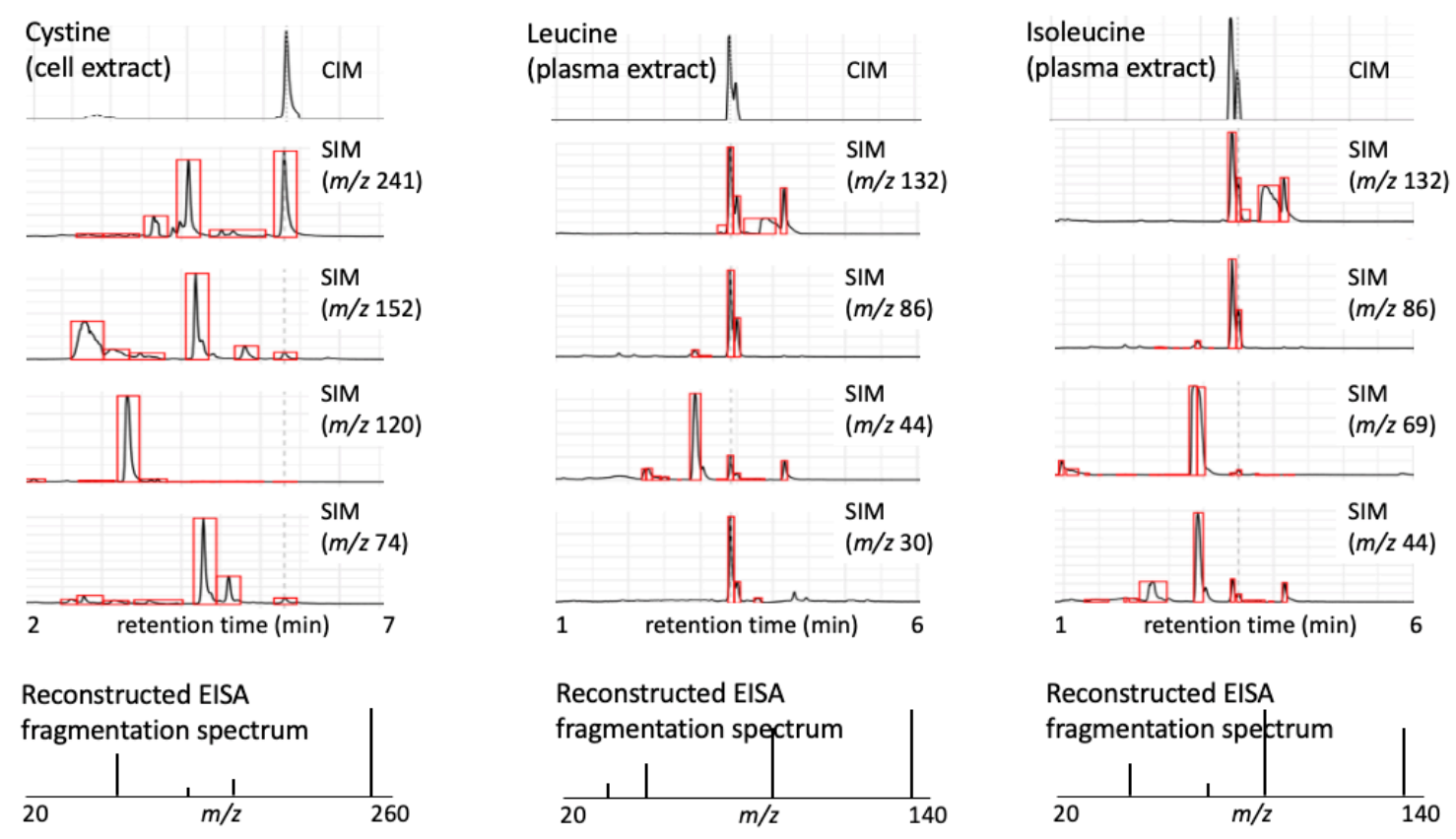

Figure 4. Correlated Ion Monitoring (CIM) of Q-MRM data. Q-MRM analysis with CIM of cystine (cell extract), leucine (plasma extract), and isoleucine (plasma extract) correlates the precursor and multiple fragment ions to generate a CIM chromatogram. The data was peak picked, aligned and threshold filtered to provide deconvolution of the Q-MRM data. CIM also evaluates the ion ratios to monitor if they match the expected (p:f1:f2:f3) ratio, if not the molecule is flagged as being contaminated with co-eluting species. A possible correction for this would be to eliminate the contaminating ion as a quantifier. Additionally, reconstructed Q-MRM fragment spectra are generated allowing for continuous monitoring of ion ratios and matching against genuine standards. p: precursor ion; f1, f2 and f3: fragment ion 1, 2, and 3, respectively.

\section{Applications and Limitations}

Typically, additional MRM transitions must be monitored with a triple quadrupole instrument to enable the identification of quantified compounds, Q-MRM adds this function to the single quadrupole mass spectrometer. Thus, Q-MRM can significantly broaden the applications of single quadrupole mass spectrometers in quantitative analysis, especially those used for routine analysis. For example, Q-MRM can be used for fragment ion monitoring which is performed daily in synthetic chemistry laboratories ${ }^{26}$. While high resolution and high detection sensitivity are vital features for structure elucidation and trace analysis, fragment ion monitoring typically requires only unit mass resolution to provide verification of product formation and to track the comings and goings of reaction starting materials, products and intermediates ${ }^{26}$. Further, due to high-maintenance equipment and complicated data analysis, a mass spectrometer (MS) is not widely used in quality control (QC) laboratories in the industry, e.g., pharmaceutical and food industry ${ }^{26}$. However, Q-MRM can help increase the application of MS-based methods in these QC laboratories, which are normally more sensitive and can substantially reduce time and cost. For example, Q-MRM can be developed to monitor the post-translational modifications (PTMs) in complex protein drugs ${ }^{26}$. Xu et al. have reported a single quadrupole method for PTMs detection and quantitation in a therapeutic monoclonal 
antibody $^{27}$. Q-MRM can also be used in the identification and quantitation of active ingredients, impurities and degradation products in the development of chemical products such as pharmaceuticals and agrochemcials $^{26}$.

However, in Q-MRM analysis, highly co-eluted analytes with precursors and fragment ion masses similar to that of the target compound can result in 'contaminated' transitions, which can lead to false positive identifications or imprecise quantification. Thus, characteristic molecular ions need to be determined for the co-eluted target analytes using either tandem mass spectra and retention time databases such as METLIN or comparing the signal intensity (peak area) ratios across standards and samples as discussed earlier. Further, QC samples can be included to the sample sequence by adding a specified amount of analyte standard to the test sample. By comparing the peak intensity (area) ratio between QC and test samples, molecular ions specific to the target analytes can be determined. If these QC protocols do not perform with sufficient accuracy, an extended liquid chromatography gradient is needed to help Q-MRM improve selectivity, especially for those closely eluted compounds with fragments or molecular ions sharing the same nominal masses.

Other ramifications of Q-MRM are that it will expand the utility of related instrumentation (e.g., QTOF and ion mobility). QTOF instrumentation can now be used both as a quantitative tool, targeted identification, and for full scan analyses. For example, the TOF could be used for un-targeted analyses, the QTOF for identification, and the $\mathrm{Q}$ for targeted quantitative validation. The combination of which are unique to QTOF technology since Orbitrap instrumentation with parallel reaction monitoring (PRM) do not allow for high sensitivity quadrupole ion selection analyses. In addition, ion mobility technology (Figure S3) could be used as an alternative discriminating feature to decipher (for example) co-eluting species.

\section{Conclusions}

Q-MRM and correlated ion monitoring (CIM) with a single quadrupole mass spectrometer offers an alternative fragmentation and quantitative analysis strategy (as illustrated in Figures 1 and 4) representing the next chapter in the development of quantitative tandem mass spectrometry and QqQ-MRM analysis. The CIM algorithm enables these analyses by autonomously processing, aligning, filtering, and compiling multiple ions providing for both precursor and in-source fragment ions to be correlated within a single chromatogram, also enabling the detection of co-eluting species based on precursor and fragment ion ratios. This utilization of this existing platform facilitates high dynamic range, selectivity, accuracy, broad availability, and reproducibility, which can be deployed on complex matrices ${ }^{10}$ and on multiple mass spectrometer types (e.g., single quadrupole, QqQ, QTOF, and Q-Orbitrap). This concept is potentially useful as one could utilize the same mass spectrometer (QTOF and Q-Orbitrap), without altering conditions, to 
perform both full scan and MRM quantitative analyses. Moreover, Q-MRM can be coupled with other separation technologies including ultra-high resolution capillary electrophoresis, ion mobility (Figure S3), and gas chromatography to further enhance their sensitivity. EISA could also provide for pseudo-MS ${ }^{3}$ and be particularly useful for the quantification of modified complex molecules such as oxidized phospholipids ${ }^{28}$ or closely related eicosanoids ${ }^{29}$. Overall, Q-MRM with single quadrupole instrumentation offers quantitative analyses for a broad range of molecules on ubiquitous instrumentation; instruments that are generally inexpensive, easy to operate, and technically less complex.

\section{REFERENCES}

1. Yost, R. A. \& Enke, C. G. Triple quadrupole mass spectrometry for direct mixture analysis and structure elucidation. Anal. Chem. 51, 1251-1264 (1979).

2. Yost, R.A. \& Enke, C.G. Selected ion fragmentation with a tandem quadrupole mass spectrometer. J. Am. Chem. Soc. 100, 2274-2275 (1978).

3. Fenn, J. B. et al. Electrospray ionization for mass spectrometry of large biomolecules. Science 246, 64-71 (1989).

4. Yuan, M., Breitkopf, S. B., Yang, X., \& Asara, J. M. A positive/negative ion-switching, targeted mass spectrometry-based metabolomics platform for bodily fluids, cells, and fresh and fixed tissue. Nat. Protoc. 7, 872-881 (2012).

5. Addona, T. A. et al. Multi-site assessment of the precision and reproducibility of multiple reaction monitoring-based measurements of proteins in plasma. Nat. Biotechnol. 27, 633-641 (2009).

6. Wang, M. et al. Mass spectrometry searches using MASST. Nat. Biotechnol. 38, 23-26 (2020).

7. Venable, J. et al. Automated approach for quantitative analysis of complex peptide mixtures from tandem mass spectra. Nat. Methods 1, 39-45 (2004).

8. Glish, G. \& Vachet, R. The basics of mass spectrometry in the twenty-first century. Nat. Rev. Drug Disco. 2, 140-150 (2003).

9. Mueller, C.A. et al. Development of a multi-target screening analysis for 301 drugs using a QTrap liquid chromatography/tandem mass spectrometry system and automated library searching. Rapid Commun. Mass Spectrom. 19, 1332-1338 (2005).

10. Domingo-Almenara, X. et al. XCMS-MRM and METLIN-MRM: a cloud library and public resource for targeted analysis of small molecules. Nat. Methods 15, 681-684 (2018).

11. Domingo-Almenara, X. et al. Autonomous METLIN-guided in-source fragment annotation for untargeted metabolomics. Anal. Chem. 91, 3246-3253 (2019).

12. Xue, J. et al. Enhanced in-source fragmentation annotation enables novel data independent acquisition and autonomous METLIN molecular identification. Anal. Chem. 92, 6051-6059 (2020).

13. Seitzer, P.M., Searle, B.C. Incorporating In-Source Fragment Information Improves Metabolite Identification Accuracy in Untargeted LC-MS Data Sets. J. Proteome Res. 18(2), 791-6.

14. Müller, C., Junker, J., Bracher, F., \& Giera, M. A gas chromatography-mass spectrometry-based whole-cell screening assay for target identification in distal cholesterol biosynthesis. Nat. Protoc. 14, 2546-2570 (2019).

15. Commission Decision (2002/657/EC) of 12 August 2002. Implementing Council Directive (96/23/EC) concerning the performance of analytical methods and the interpretation of results. Off J Eur Communities 2002, L221: 8-36.

16. United States Food and Drug Administration (2018). Bioanalytical method validation guide for industry. https://www.fda.gov/media/70858/download (accessed on August 2020) 
17. Matuszewski, B. K., Constanzer, M. L., \& Chavez-Eng, C. M. Strategies for the assessment of matrix effect in quantitative bioanalytical methods based on HPLC-MS/MS. Anal. Chem. 75, 3019-3030 (2003).

18. Ivanisevic, J. et al. Arteriovenous Blood Metabolomics: A Readout of Intra-Tissue Metabostasis. Sci. Rep. 5, 12757 (2015).

19. Serafim, V. et al. Development and Validation of a LC-MS/MS-Based Assay for Quantification of Free and Total Omega 3 and 6 Fatty Acids from Human Plasma. Molecules 24, 360-361 (2019).

20. Beyer, B. et al. Metabolomics-based discovery of a metabolite that enhances oligodendrocyte maturation. Nat. Chem. Biol. 14, 22-28 (2018).

21. Domingo, X. et al. The METLIN small molecule dataset for machine learning-based retention time prediction. Nat. Commun. 10, 5811-5819 (2019).

22. Xue, J. et al. METLIN $\mathrm{MS}^{2}$ molecular standards database: a broad chemical and biological resource. Nat. Methods 17, 953-954 (2020).

23. Wishart, D. S. et al. HMDB 3.0-The Human Metabolome Database in 2013. Nucleic Acids Res. 41 (Database issue), D801- D807 (2013).

24. Lisec J., Hoffmann F., Schmitt C. \& Jaeger C. Extending the Dynamic Range in Metabolomics Experiments by Automatic Correction of Peaks Exceeding the Detection Limit. Anal. Chem. 88, 7487-7492 (2016).

25. Liu, H., Lam, L. \& Dasgupta, P.K. Expanding the linear dynamic range for multiple reaction monitoring in quantitative liquid chromatography-tandem mass spectrometry utilizing natural isotopologue transitions. Talanta 87, 307-310 (2011).

26. Bu, X., Regalado, E.L., Hamilton, S.E. \& Welch, C.J. The emergence of low-cost compact mass spectrometry detectors for chromatographic analysis. Trends Anal. Chem. 82, 22-34 (2016).

27. $\mathrm{Xu}, \mathrm{W}$. et al. A Quadrupole Dalton-based multi-attribute method for product characterization, process development, and quality control of therapeutic proteins. MAbs. 9, 1186-1196 (2017).

28. Jónasdóttir, H.S. et al. Detection and structural elucidation of esterified oxylipids in human synovial fluid by electrospray ionization-fourier transform ion-cyclotron mass spectrometry and liquid chromatography-ion trap-MS(3): detection of esterified hydroxylated docosapentaenoic acid containing phospholipids. Anal. Chem. 85, 6003-6010 (2013).

29. Chakraborty, T. et al. Eicosanoid biosynthesis influences the virulence of Candida parapsilosis. Virulence. 9, 1019-1035 (2018). 